\title{
HLA-A2, A3-Restricted FGF-5 Peptides/Montanide ISA-51 Vaccine
}

National Cancer Institute

\section{Source}

National Cancer Institute. HLA-A2, A3-Restricted FGF-5 Peptides/Montanide ISA-51

Vaccine. NCl Thesaurus. Code C48394.

A peptide vaccine comprised of synthetic HLA-A2- and HLA-A3-binding peptides, derived from amino acid sequences of fibroblast growth factor-5 (FGF-5), combined with the adjuvant Montanide ISA-51 with potential antineoplastic activity. HLA-A2, A3-restricted FGF-5 peptides contain motifs recognized by the MHC class I molecules HLA-A2 and HLA-A3 and may stimulate a cytotoxic T-cell response against tumor cells that overexpress FGF-5. Montanide ISA-51, a stabilized water-in-oil emulsion adjuvant containing mineral oil with mannide oleate added as a surfactant, non-specifically stimulates cell-mediated immune responses to antigens. 